\title{
MECHANISM OF OPTICAL DETECTION OF MAGNETIC RESONANCE IN $\mathrm{Cd}_{1-x} \mathrm{Mn}_{x} \mathrm{Te}^{*}$
}

\author{
M. Godlewski, M. Surma, R.R. GalązKa \\ Institute of Physics, Polish Academy of Sciences \\ Al. Lotników 32/46, 02-668 Warszawa, Poland
}

\section{NGUYen The KHOI}

Institute of Experimental Physics, Warsaw University

Hoża 69, 00-681 Warszawa, Poland

\section{S.J.C.H.M. Gisbergen, T. Gregorkiewicz and C.A.J. AmmerlaAN}

University of Amsterdam, Amsterdam, The Netherlands

The photoluminescence and optically detected magnetic resonance studies of $\mathrm{Cd}_{1-x} \mathrm{Mn}_{x} \mathrm{Te}(x=0.095)$ are presented. The $\mathrm{Mn}^{2+}$ magnetic resonance is detected optically via the changes of "edge" emission induced by the decrease of the Mn spin system magnetization.

PACS numbers: 76.70.Hb, 75.50.Pp, 78.20.Ls

\section{Introduction}

Strong magnetic interaction affects the optical properties of semimagnetic semiconductors. This is demonstrated in the present work by applying the optically detected magnetic resonance (ODMR) technique. The changes of the "edge" emission of $\mathrm{Cd}_{1-x} \mathrm{Mn}_{x} \mathrm{Te}(x=0.095)$ induced by the $\mathrm{Mn}^{2+}$ resonance are studied. We show that the photoluminescence (PL) changes have the resonant character, which allows us to conclude on the ODMR detection mechanism.

\section{Experimental results}

The PL spectrum of the $\mathrm{Cd}_{1-x} \mathrm{Mn}_{x}$ Te sample is shown in Fig. 1a together with the reflectivity spectrum measured in order to determine the Mn mole fraction in the crystal studied. The PL spectrum consists of the free exciton (FE) line at $1.738 \mathrm{meV}$, the acceptor bound exciton (ABE) line at $1.718 \mathrm{meV}$ and a broad PL band at $1.667 \mathrm{meV}$, which is due to free electron-acceptor (FA) and/or donor-acceptor pair (DAP) transition. In Fig. 1b we show the PL spectrum measured with magnetic field set at $1.3 \mathrm{~T}$. All emission lines shift towards lower energies with an increase of the external magnetic field. The intensity ratio between $\mathrm{FE}$ to ABE

*This work was partly supported by the grant no. 204699101 of the Committee for Scientific Research.

tOn leave from Hanoi Pedagogical University, Hanoi, Vietnam. 
and $A B E$ to FA/DAP is also changed. The intensity of the ABE line is reduced, as observed previously $[1,2]$. The temperature dependence of the PL spectrum was also measured to exclude the possibility that the PL changes observed in the ODMR experiments are due to the sample heating by the applied microwave power.

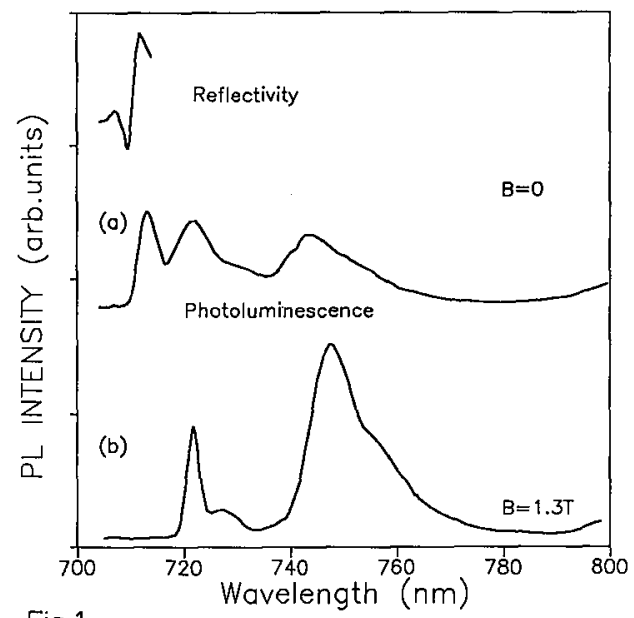

Fig.1

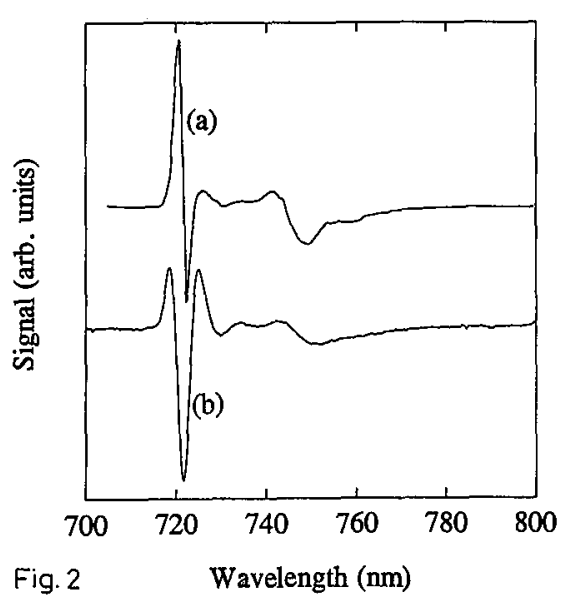

Fig. 2

Fig. 1. Photoluminescence and reflectivity spectrum of the $\mathrm{Cd}_{1-x} \mathrm{Mn}_{x} \mathrm{Te}$ measured at $2 \mathrm{~K}$ under argon laser excitation. The spectrum shown in (b) was taken for external magnetic field set at $1.3 \mathrm{~T}$.

Fig. 2. Spectral dependence of the $\mathrm{Mn}^{2+} \mathrm{ODMR}$ signal measured as the change of the PL spectrum in phase with on-oft modulated microwaves and the external magnetic field set at the $\mathrm{Mn}^{2+}$ resonance (b). In (a) the change of the PL spectrum is shown induced by a reduction of external magnetic field. This spectrum was obtained as a difference of two PL spectra taken at $1.2 \mathrm{~T}$ and $1.3 \mathrm{~T}$.

The PL spectrum measured in phase with the on-off modulated microwave power shows a very strong $\mathrm{Mn}^{2+}$ magnetic resonance signal. This signal was observed as the change of the "edge" emission shape and intensity but also via a slight decrease of the intensity of a deep DAP emission at $1100 \mathrm{~nm}$. The signal observed in the ODMR experiment is identical with that measured in a conventional electron spin resonance (ESR) study. The PL response to the magnetic resonance (spectral dependence of ODMR) was studied in the following way: the constant light excitation was used and the relative PL changes were measured in phase with on-off modulated microwave power. Such spectral dependence of the $\mathrm{Mn}^{2+}$ resonance signal is shown in Fig. 2b, which demonstrates the response of the "edge" emission to the on-off modulated microwaves with the magnetic field set at the $\mathrm{Mn}^{2+}$ resonance. This dependence is compared with the relative change of the PL spectrum induced by about $10 \%$ decrease of the external magnetic field (Fig. 2a). 


\section{Discussion}

The mechanism of optical detection of magnetic resonances was discussed by several authors [3]. Some of the mechanism included non-resonant PL changes or changes induced by the sample heating. Malyavkin and Komarov et al. [4, 5] proposed that in semimagnetic semiconductors the sample magnetization may change at the magnetic resonance, which in turn will change energy level splitting of excitons and impurities. This would be observed in the PL experiment as a shift of the relevant PL lines and, possibly, as a change of their relative intensities.

If this mechanism is responsible for the ODMR detection the spectra shown in Fig. 2 should be similar. Such similarities are observed for the response of the $A B E$ and FA/DAP emissions to the magnetic resonance and the decrease of the magnetic field. We conclude from these data that $\mathrm{Mn}^{2+}$ resonance is detected due to the change of the sample magnetization at $\mathrm{Mn}^{2+}$ resonance. The strong $s p-d$ exchange interaction between the magnetic moments of free and bound carriers and the localized Mn spins induces a very large splitting of electronic states of excitons and impurities. This splitting is directly proportional to the magnetization of the Mn spin system induced by the external magnetic field. The $\mathrm{Mn}^{2+}$ resonance reduces the magnetization of the sample and, consequently, influences the splitting of exciton and impurity states. As a result the "edge" emission spectra shift towards higher energies which enables optical detection of the magnetic resonance. We have confirmed the above explanation by measuring the magnetic resonance signal via each separate PL line. It was observed that the PL changes are induced by the $\mathrm{Mn}^{2+}$ resonance and that no non-resonant signals occur. We could also definitely reject the possibility that PL changes are induced by sample heating by the applied microwave power.

The decrease of the sample magnetization does not explain the whole response of the "edge" emission to the magnetic resonance. The free exciton emission seems to be nearly symmetrically broadened at the magnetic resonance which is not fully understood at present. Such a response is different from that predicted by only the change of the Mn spin system magnetization. We also indicate that we can exclude the effects of sample heating. The strong broadening of the FE line was not observed in our studies of the temperature dependence of the PL spectrum.

\section{Acknowledgments}

The authors are indebted to Drs. J.A. Gaj and Z. Wilamowski for helpful comments.

\section{References}

[1] R.Y Tao, M.M. Moriwaki, W.M. Becker, R.R. Gałazka, J. Appl. Phys. 53, 3772 (1982).

[2] M.M. Moriwaki, W.M. Becker, R.R. Gałazka, Phys. Rev. B 26, 3165 (1982).

[3] B.C. Cavenett, Adv. Phys. 30, 475 (1981).

[4] A.V. Malyavkin, Phys. Status Solidi B 115, 353 (1983).

[5] A.V.Komarov, S.M. Ryabchenko, O.V. Terletskii, I.I. Zheru, R.D. Ivanchuk, Sov. Phys. JETP 46, 318 (1977). 